\title{
Mitral Valve Repair for Barlow's Disease with Mitral Annular and Subvalvular Calcification: A Case Report
}

\author{
Kosuke Nakamae, M.D., Takashi Oshitomi, M.D., Kentaro Takaji, M.D., Hideyuki Uesugi, M.D. \\ Division of Cardiovascular Surgery, Saiseikai Kumamoto Hospital, Kumamoto, Japan
}

\section{ARTICLE INFO}

Received September 27, 2021

Accepted November 26, 2021

Corresponding author

Kosuke Nakamae

Tel 82-96-351-8000

Fax 82-96-326-3045

E-mail kn_beat_maxplus@yahoo.co.jp ORCID

https://orcid.org/0000-0003-3848-0153

\begin{abstract}
Barlow's disease with mitral annular calcification encompassing the subvalvular apparatus, including the valve leaflet and chordae, is extremely rare, and mitral valve repair in such cases is challenging. We report a case of a 60-year-old woman with mitral valve regurgitation that was successfully controlled by resecting the rough zone of P2 and calcifications on the excess leaflet regions and subvalvular apparatus, while retaining the calcification of P3 and implanting artificial chordae and an annuloplasty ring. Mitral valve repair for such cases requires an individualized and compounded surgical strategy for the technique to treat Barlow's disease and manage calcification to control mitral regurgitation.
\end{abstract}

Keywords: Barlow's disease, Decalcification, Heart valve diseases, Mitral valve repair, Case report

\section{Case report}

Text Barlow's disease (BD), which is known to involve leaflet myxoid degeneration (leaflet thickening, large redundant leaflets, chordal elongation or rupture, and annular dilation), sometimes causes mitral regurgitation (MR) [1]. Although some factors correlate with the prevalence of mitral annular calcification (MAC) [2], BD with MAC encompassing the subvalvular apparatus is rare, and surgical strategies for mitral valve repair (MVr) remain unclear. In this case report, we present a case which we successfully controlled mitral valve (MV) regurgitation by resecting the valve leaflet, performing decalcification, and retaining a part of the calcification.

A 60-year-old woman was referred to Saiseikai Kumamoto Hospital with dyspnea on exertion. Echocardiography revealed cardiac enlargement, no asynergy of left ventricular wall motion, an ejection fraction (EF) of $65 \%$, severe MR (effective regurgitation orifice area of $0.59 \mathrm{~cm}^{2}$ ) with bi-leaflet thickening (Fig. 1A), myxomatous changes with excessive tissue formation (Fig. 1B), and billowing with prolapse (Fig. 1C), which are characteristic pathologic changes of BD. Computed tomography (CT) revealed calcification of the mitral annulus, leaflets, and subvalvular apparatus, such as the chordae (Fig. 1D). Our cardiology team recommended surgery based on the severity of MR.

Surgical procedure is as follows (Supplementary Video 1). Surgery was performed with the da Vinci surgical system (Intuitive Surgical Inc., Sunnyvale, CA, USA) using 4 chest ports, and cardiopulmonary bypass was initiated using the right femoral artery and vein. Cardiac standstill was obtained from antegrade cardioplegia, and we approached the MV through a right-side left atriotomy. The bi-leaflet of the MV was large and excessively thickened; annular, leaflet, and subvalvular calcifications at $\mathrm{P} 2$ and $\mathrm{P} 3$ were observed. We resected the rough zone of $\mathrm{P} 2$ in a pentagonal shape, and performed decalcification by en bloc resection of the calcium deposits at the leaflet and chordae of P2. The calcification of the P3 leaflet was retained. After suturing the P2 leaflet in an inverted T-shape, we implanted 2 artificial chordae from the bilateral papillary muscles and a 36-mm Physio Flex annuloplasty ring (Edwards Lifesciences, Irvine, CA, USA). MV competence upon normal saline injection was satisfactory. The operative, cardiopulmonary bypass, and cross-clamp times were 359 minutes, 277 minutes, and 200 minutes, respectively.

The postoperative course was uneventful. At 1 week postoperatively, echocardiography showed preserved EF (59\%), improvement of the left ventricular endo-diastolic diameter from $55 \mathrm{~mm}$ preoperatively to $45 \mathrm{~mm}$, with a 

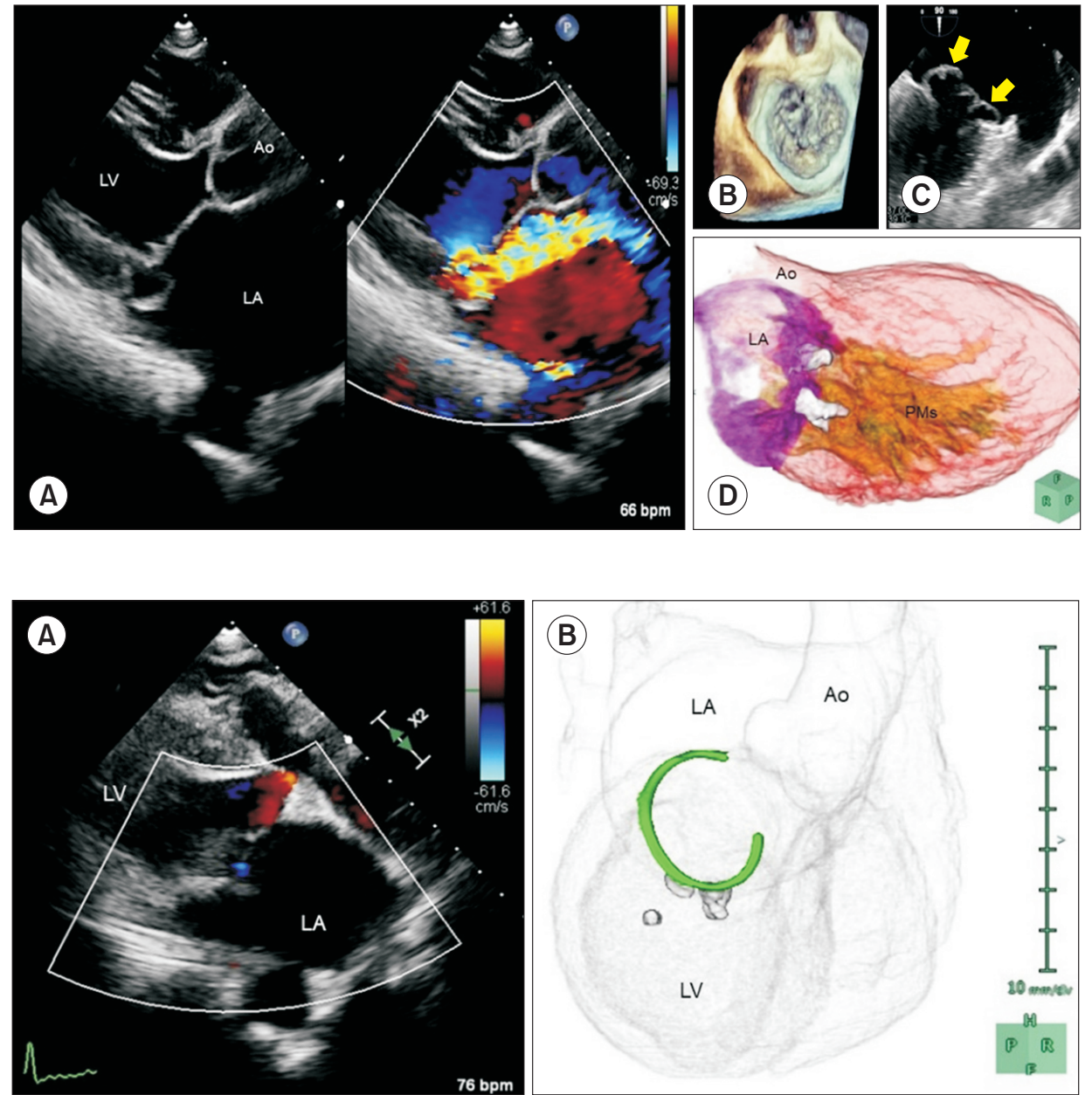

Fig. 1. (A) Preoperative echocardiography reveals severe mitral regurgitation. Bi-leaflet thickening, (B) myxomatous change, and (C) bi-leaflet prolapse (yellow arrow), indicating Barlow's disease. (D) Preoperative computed tomography reveals calcification of mitral annular and subvalvular apparatus at P2 and P3. Ao, aorta; LA, left atrium; LV, left ventricle; PM, papillary muscle. mean pressure gradient of $2.2 \mathrm{~mm} \mathrm{Hg}$, and no residual $\mathrm{MR}$ (Fig. 2A). Enhanced CT performed at 1 week postoperatively revealed resection of most calcification in the region of P2, but calcification in P3 were retained (Fig. 2B). At a 1-year follow-up visit, progression of MR and cardiac enlargement were not observed.

Our institutional review board made the decision that ethical approval was not needed for this study. Informed consent was obtained from the patient.

\section{Discussion}

The prevalence of MAC in patients undergoing MVr is relatively high. Fusini et al. [2] reported that some factors correlated with MAC and that posterior annular involvement was the most common site of MAC. However, Carpentier et al. [3] reported that cases of MAC involving the subvalvular apparatus, such as chordae and papillary muscles, were rare. Therefore, MVr for BD with MAC encom- passing the subvalvular apparatus is a challenging procedure, and management of calcifications, the method of $\mathrm{MVr}$, and whether to repair or replace it are the key surgical considerations.

Various methods have been previously reported regarding the management of MAC. Carpentier et al. [3] reported en bloc resection and reconstruction of the atrioventricular groove with 2-0 vertical sutures. Loulmet et al. [4] reported the modified patch technique for repairing an atrioventricular groove after en bloc resection of extensive MAC. Residual MAC might limit the leaflet mobility and cause residual MR or postoperative mitral stenosis (MS); however, excessive resection may also cause disruption of the morphology of the MV, which makes it impossible to repair. In this case, calcification involved the leaflet and chordae of P2 and P3, as well as the annulus of P3. P2 calcification contributed to restrained movement of the leaflet and may have caused postoperative MS. Conversely, the P3 calcification involved a smaller area and might not have contribut- 
ed to postoperative MS; however, its resection may have collapsed the MV morphology.

Many reports have investigated the methods of MVr for BD. Ben Zekry et al. [5] analyzed simple repair for BD with only an annuloplasty ring; however, this seems incomplete because it included a certain number of cases that had systolic anterior motion (SAM) of the anterior leaflet. Some reports have suggested a relationship between $\mathrm{BD}$ and mitral annular disjunction, and Hiemstra et al. [6] reported annular remodeling and stabilization as being important for the surgical treatment in $\mathrm{BD}$ in order to correct the dilation and abnormal annulus movement. Miura et al. [7] reported a restoration technique that involved reducing the lateral volume of excess leaflets in the rough zone and correcting the prolapse using a few artificial chordae. Therefore, we followed the MVr strategy according to these concepts of fixing the MV by annuloplasty, reducing excess volume by resection, and correcting the prolapse with artificial chordae, and we controlled MR without causing residual MR, MS, or SAM.

In conclusion, $\mathrm{MVr}$ for BD with MAC encompassing the subvalvular apparatus requires careful assessment of the distribution of calcifications, valve morphology, and surgical techniques for managing calcification.

\section{Conflict of interest}

No potential conflict of interest relevant to this article was reported.

\section{ORCID}

Kosuke Nakamae: https://orcid.org/0000-0003-3848-0153 Takashi Oshitomi: https://orcid.org/0000-0001-8942-0981 Kentaro Takaji: https://orcid.org/0000-0001-9336-3860 Hideyuki Uesugi: https://orcid.org/0000-0002-6214-1373

\section{Supplementary materials}

Supplementary materials can be found via https://doi. org/10.5090/jcs.21.113. Supplementary Video 1. Intraoperative mitral valve repair technique for Barlow's disease with mitral annular calcification encompassing the subvalvular apparatus.

\section{References}

1. Barlow JB, Bosman CK. Aneurysmal protrusion of the posterior leaflet of the mitral valve: an auscultatory-electrocardiographic syndrome. Am Heart J 1966;71:166-78.

2. Fusini L, Ghulam Ali S, Tamborini G, et al. Prevalence of calcification of the mitral valve annulus in patients undergoing surgical repair of mitral valve prolapse. Am J Cardiol 2014;113:1867-73.

3. Carpentier AF, Pellerin M, Fuzellier JF, Relland JY. Extensive calcification of the mitral valve anulus: pathology and surgical management. J Thorac Cardiovasc Surg 1996;111:718-30.

4. Loulmet DF, Ranganath NK, Neragi-Miandoab S, Koeckert MS, Galloway AC, Grossi EA. Advanced experience allows robotic mitral valve repair in the presence of extensive mitral annular calcification. J Thorac Cardiovasc Surg 2021;161:80-8.

5. Ben Zekry S, Spiegelstein D, Sternik L, et al. Simple repair approach for mitral regurgitation in Barlow disease. J Thorac Cardiovasc Surg 2015;150:1071-7.

6. Hiemstra YL, Tomsic A, Gripari P, et al. Evolution from mitral annular dysfunction to severe mitral regurgitation in Barlow's disease. Interact Cardiovasc Thorac Surg 2021;32:506-14.

7. Miura T, Ariyoshi T, Tanigawa K, et al. Technical aspects of mitral valve repair in Barlow's valve with prolapse of both leaflets: triangular resection for excess tissue, sophisticated chordal replacement, and their combination (the restoration technique). Gen Thorac Cardiovasc Surg 2015;63:61-70. 